\title{
Mathematical model of a fountain with a water picture in the shape of an hourglass
}

\author{
Kazimierz Peszynski ${ }^{1, *}$, Daniel Perczynski ${ }^{1}$, and Lidia Piwecka $^{1}$ \\ ${ }^{1}$ UTP University of Science and Technology, Al. prof. S. Kaliskiego 7, 85-796 Bydgoszcz, Poland
}

\begin{abstract}
The work presents a mathematical model of a fountain providing an hourglass-shaped water image based on four of its basic parameters: height $H$, coefficient of contraction $\chi$, diameter of $n$ nozzles arrangement $D_{\text {na }}$, and coefficient of contraction location $\sigma$. The developed model makes possible to determine the angle $\alpha$ specifying the slope of the nozzles to the horizontal plane and determining the remaining parameters of the fountain, especially its outer diameter $D_{\mathrm{f}}$ as well as the diameter of the position of the stream tops $D_{\max }$. The developed model has been implemented in an Excel spreadsheet, which enables quick conversion of desired water images. The developed model works for $n \leq 24$ nozzles, however, derived dependencies allow for a quick extension of the model for $n>24$.
\end{abstract}

\section{Nomenclature \\ A $\quad \mathrm{m}^{2} \quad$ area \\ $d, D \quad \mathrm{~m} \quad$ diameter \\ $g \quad \mathrm{~m} \cdot \mathrm{s}^{-2}$ gravitational acceleration \\ $H \quad \mathrm{~m}$ height \\ $h \quad \mathrm{~m} \quad$ vertical coordinate of jet path \\ $l \quad \mathrm{~m} \quad$ horizontal coordinate of jet path \\ $L \quad \mathrm{~m} \quad$ jet horizontal range \\ $n \quad-\quad$ the number of nozzles in the assembly \\ $t \quad \mathrm{~s}$ time \\ $v \quad \mathrm{~m} \cdot \mathrm{s}^{-1}$ velocity \\ $i \quad \mathrm{~m}^{3} \mathrm{~s}^{-1} \quad$ volume flow rate \\ $\alpha \quad \mathrm{rad} \quad$ the angle of the nozzle inclination to the level

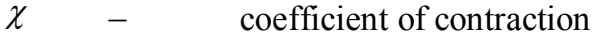 \\ $\gamma \quad \mathrm{rad}$ the angle of deviation of the trace of the stream flow surface from the straight line between centre point and $1^{\text {st }}$ nozzle \\ $\sigma \quad-\quad$ coefficient of contraction location \\ Indexes

$\begin{array}{ll}\mathrm{c} & \text { contraction } \\ \mathrm{max} & \text { maximum value, e.g. height, range } \\ \mathrm{n} & \text { nozzle } \\ \mathrm{na} & \text { nozzle assembly } \\ 0 & \text { initial value }\end{array}$

\section{Introduction}

Fountains for many centuries have been enriched by landscapes of castle parks, towns and rural areas. Their producers, as part of the competition, constantly introduce innovative improvements in the form of the variability of the water image, light effects and musical effects. Control of such complex facilities requires the use of modern computational techniques, usually PLC controllers. In turn, programming the controllers requires a mathematical model. The development of a mathematical model providing an hourglass-shaped water image is the subject of this paper.

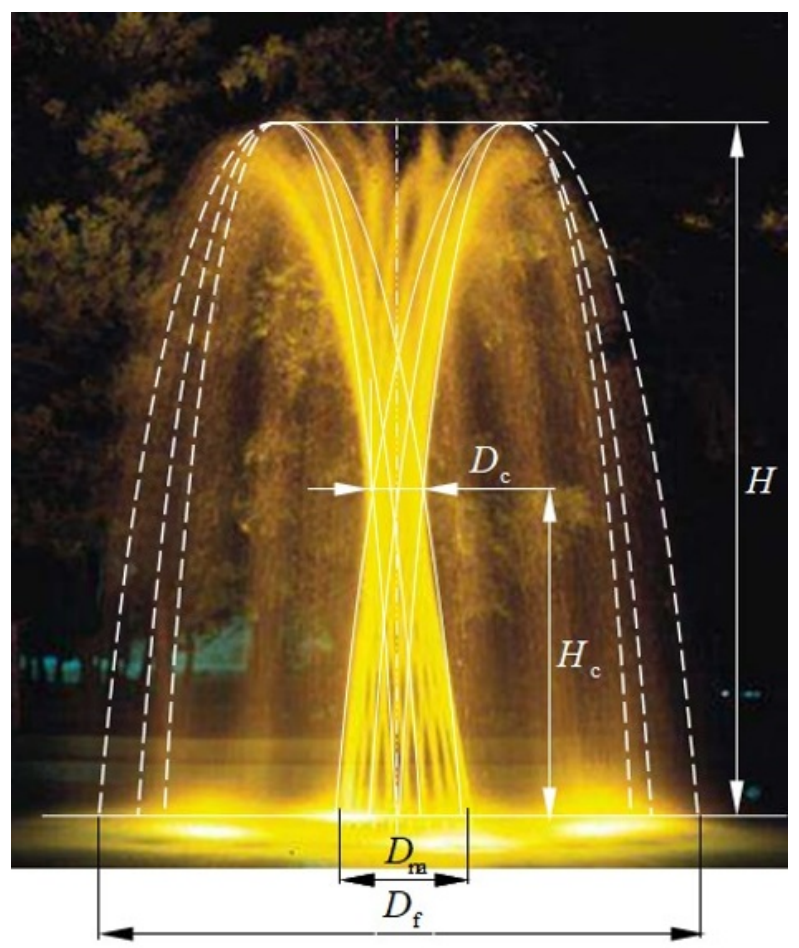

Fig. 1. Basic geometric parameters of the designed fountain. 
Fig. 1 shows the water image of the analysed fountain and its basic geometrical parameters: fountain height $H_{\max }$, nozzle assembly diameter $D_{\text {na }}$, virtual contraction diameter of all jets $D_{\mathrm{c}}$, distance from the base of the fountain $H_{\mathrm{c}}$. Diameter of the fountain $D_{\mathrm{f}}$ is a parameter resulting from the setting of the above parameters.

\section{Mathematical model}

\subsection{Path of free water jet}

Outlet jet of water flows from nozzle of diameter $d_{\mathrm{n}}$ at an angle $\alpha$ to the horizontal level with the initial speed $v_{0}$. The initial velocity is treated as the average velocity over the entire nozzle cross-section, so that the product of this velocity and the transverse cross-section of the nozzle determines the volume flow rthrough one nozzle

$$
i_{\mathrm{n}} \quad \sim_{\mathrm{n} \cdot \mathrm{v}} \mathrm{m}^{3} \cdot \mathrm{s}^{-1},
$$

therefore the equation

$$
i_{\mathrm{na}} \quad \cdots \cdot \mathrm{n} \cdot \mathrm{v} \quad \mathrm{m}^{3} \cdot \mathrm{s}^{-1},
$$

determines the total flow rate through all the fountain nozzles.

The vector of the outgoing water velocity has two component vectors $v_{0 \mathrm{r}}$ and $v_{0 \mathrm{~h}}$ (Fig. 1) of values $v_{0 l}=v_{0} \cos \alpha$ and $v_{0 \mathrm{~h}}=v_{0} \sin \alpha$.
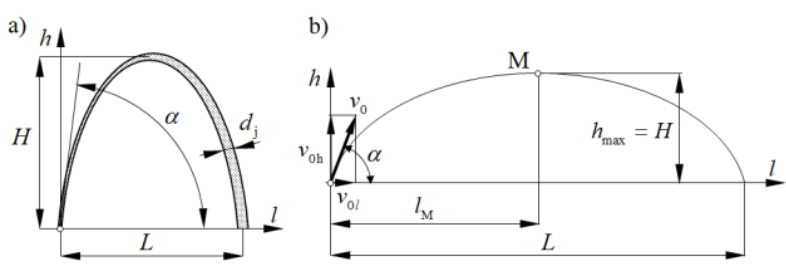

Fig. 2. Jet outgoing from nozzle a) throw with an angle to horizontal plane, b) schematic for rough analysis

Water jet motion (its volume element) is the resultant motion of two straight-line movements [1]: uniform rectilinear with initial velocity $v_{1}=v_{01}=v_{0} \cos \alpha$ in the horizontal direction and non uniform rectilinear with initial velocity $v_{0 \mathrm{~h}}=v_{0} \sin \alpha$ in vertical direction. Therefore after the time $t$ from the moment the traffic starts the observed water particle had travelled in a horizontal direction

$$
l=\left(v_{0} \cos \alpha\right) t
$$

and in the vertical direction

$$
h=\left(v_{0} \sin \alpha\right) t-\frac{g t^{2}}{2}
$$

Determining $t$ from the equation (3) and substituting into (4) we obtain the equation of trajectory of the jet

$$
h=\frac{l \sin \alpha}{\cos \alpha}-\frac{g l^{2}}{2 v_{0}^{2} \cos ^{2} \alpha}
$$

This is a quadratic equation. Its graph, and hence the path of movement of the jet outcoming from the nozzle inclined at an angle $\alpha$ to the horizontal level is a parabola.

Horizontal range of the jet we obtain after substituting to path trajectory value of (for simplicity, we assume that the bottom of the fountain is horizontal)

$$
0=\frac{L \sin \alpha}{\cos \alpha}-\frac{g L^{2}}{2 v_{0}^{2} \cos ^{2} \alpha} \text { or } \frac{L\left(v_{0}^{2} \sin 2 \alpha-g L\right)}{2 v_{0}^{2} \cos ^{2} \alpha}=0
$$

Comparing nominator of equation (6) to zero, we obtain $L\left(g L-v_{0}^{2} \sin 2 \alpha\right)=0$. This means that either $L=0$, or the expression in brackets is zero. The first condition means the point from which the jet flows out, whereas means the point, where the jet drops. Therefore is a correct relationship $g L-2 v_{0}^{2} \sin 2 \alpha=0$, so the range of the fountain $l=L$ is

$$
L=\frac{v_{0}^{2} \sin 2 \alpha}{g}
$$

When designing fountains one of the basic parameters of the output is the maximum height to which the water jet reaches. This maximum height of the jet, we obtain by comparing to zero first derivative of the function $h=f(l)$ defined by the formula (5). Therefore

$$
\frac{\mathrm{d} h}{\mathrm{~d} l}=\frac{-2 g}{2 v_{0}^{2} \cos ^{2} \alpha} l+\frac{\sin \alpha}{\cos \alpha}=0
$$

whence

$$
l_{\mathrm{M}}=\frac{2 v_{0}^{2} \sin \alpha \cos \alpha}{2 g}=\frac{v_{0}^{2} \sin 2 \alpha}{2 g}=0,5 L
$$

As is clear from comparison with formula (7) the maximum height is in the middle of the jet range. After substitution of the obtained value $l_{\mathrm{M}}=0,5 \mathrm{~L}$ to the path equation (5), we obtain formula which specifies the maximum height of the water stream

$$
h_{\max }=H=\frac{v_{0}^{2} \sin ^{2} \alpha}{2 g}
$$

\subsection{Outflow geometry for the fountain image of hourglass type.}

Water image of fountain in a hourglass shape can be determined on the basis of theoretical analysis of the spatial geometric relationships. It is assumed that it is a boundary of streams flowing from $n$ nozzles of inner diameter $d_{\mathrm{n}}$ distributed evenly on a circle of diameter $D_{\text {na }}$. The primary task was to find a mathematical model of analysed fountain, based on which is set the inclination angle $\alpha$ of the nozzle to the horizontal plane. For analysis generalization were introduced dimensionless parameters:

- coefficient of fountain contraction

$$
\chi=\frac{D_{\mathrm{c}}}{D_{\mathrm{n}}}
$$

- coefficient of fountain contraction location 


$$
\sigma=\frac{h_{\mathrm{c}}}{h_{\max }}
$$

In the light of formulas (11) and (12) we can write that $D_{\mathrm{c}}=\chi D_{\text {na }}$ and $h_{\mathrm{c}}=\sigma h_{\text {max }}$.

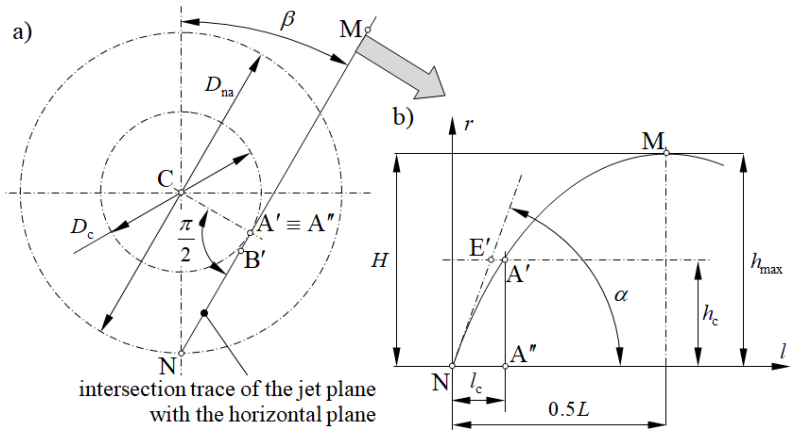

Fig. 3. Geometry of one jet: a) horizontal projection, b) projection on jet plane

Design parameters defined in this way define the basic parameters of the fountain water image. The jet plane jet is deflected from the plane passing through the axis of the fountain lying at point $\mathrm{C}$ and the point $\mathrm{N}$ by an angle of $\beta$. Angle $\beta$ can be determined from rectangular triangle $\mathrm{CA}^{\prime \prime} \mathrm{N}$ (Fig. 3a):

$$
\sin \beta=\frac{D_{\mathrm{c}}}{D_{\mathrm{na}}}=\frac{\chi D_{\mathrm{na}}}{D_{\mathrm{na}}}=\chi \text { or } \beta=\arcsin \chi
$$

In subsection 2.1 path of jet outgoing from nozzle (5) were determined. This jet should pass through the point $\mathrm{A}^{\prime}\left(l_{\mathrm{c}} ; h_{\mathrm{c}}\right)$. From a mathematical point of view, this means that it must satisfy the equation of the jet path for the coordinates of this point (Fig. 3b).

After substituting $l_{\mathrm{c}}$ and $h_{\mathrm{c}}$ into equation of trajectory we get

$$
h_{\mathrm{c}}=\frac{l_{\mathrm{c}} \sin \alpha}{\cos \alpha}-\frac{g l_{\mathrm{c}}^{2}}{2 v_{0}^{2} \cos ^{2} \alpha}
$$

From equation (14) we can determine the slope angle $\alpha$ between the nozzle axis and horizontal level, however, it is necessary to know the initial velocity $v_{0}$ of outgoing jet. This speed can be counted from the equation (10)

$$
h_{\max }=\frac{v_{0}^{2} \sin ^{2} \alpha}{2 g} \rightarrow v_{0}^{2}=\frac{2 g h_{\max }}{\sin ^{2} \alpha}
$$

After substituting for $v_{0}^{2}$ to (14) and using the geometric formula $l_{\mathrm{c}}=0.5 D_{\mathrm{n}} \cos \beta=0.5 D_{\mathrm{n}} \sqrt{1-\chi^{2}}$ (Fig. 3a) and $h_{\mathrm{c}}=\sigma h_{\max }$ we get

$$
\begin{aligned}
& 0.25 D_{\text {na }}^{2}\left(1-\chi^{2}\right) \tan ^{2} \alpha+ \\
& -2 D_{\text {na }} h_{\max } \sqrt{1-\chi^{2}} \tan \alpha+4 \sigma h_{\max }^{2}=0
\end{aligned}
$$

This is a quadratic equation, so you can expect two angles: $\alpha_{1}$ for an ascending jet and $\alpha_{2}$ for falling jet.

This may be solved by using the formula:

$$
\tan \alpha_{1,2}=\frac{-b \pm \sqrt{b^{2}-4 a c}}{2 a}
$$

where from equation (16): $a=0.25 D_{\text {na }}^{2}\left(1-\chi^{2}\right)$,

$$
b=-2 D_{\text {na }} h_{\max } \sqrt{1-\chi^{2}} \text {, and } c=4 \sigma h_{\max }^{2} .
$$

If

$$
\begin{aligned}
\Delta & =b^{2}-4 a c \\
& =\left(2 D_{\text {na }} h_{\max } \sqrt{1-\chi^{2}}\right)^{2}-4 \cdot 0.25 D_{\mathrm{n}}^{2}\left(1-\chi^{2}\right) 4 \sigma h_{\max }^{2} \\
& =4 D_{\text {na }}^{2}\left(1-\chi^{2}\right)(1-\sigma) h_{\max }^{2}
\end{aligned}
$$

hence

$$
\begin{aligned}
\tan \alpha_{1,2} & =\frac{2 D_{\text {na }} h_{\max } \sqrt{1-\chi^{2}} \pm \sqrt{\Delta}}{2 \cdot 0.25 D_{\text {na }}^{2}\left(1-\chi^{2}\right)} \\
& =\frac{4(1 \pm \sqrt{1-\sigma}) h_{\text {max }}}{D_{\text {na }} \sqrt{1-\chi^{2}}}
\end{aligned}
$$

therefore

$$
\alpha_{1,2}=\arctan \frac{4(1 \pm \sqrt{(1-\sigma)}) h_{\text {max }}}{D_{n} \sqrt{1-\chi^{2}}} \operatorname{rad}
$$

In degrees slop of nozzle is determined by equation:

$$
\left.\alpha_{1,2}=\frac{180^{\circ}}{\pi} \arctan \frac{4(1 \pm \sqrt{(1-\sigma)}) h_{\max }}{D_{n} \sqrt{1-\chi^{2}}} \quad{ }^{\circ}\right]
$$

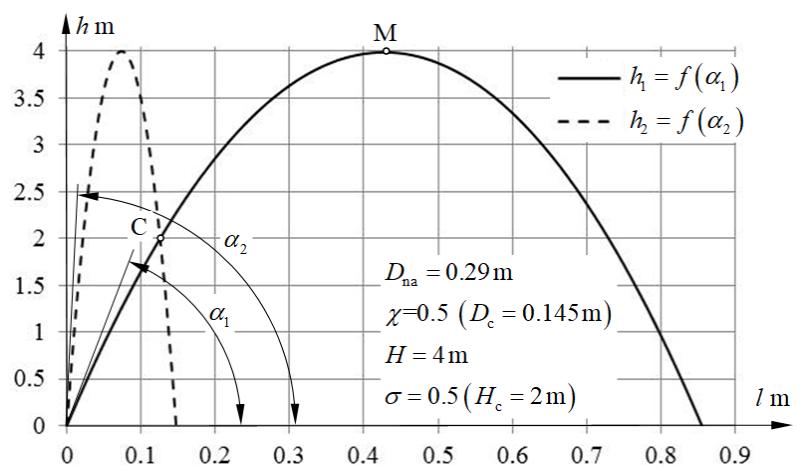

Fig. 4. Jet path on $(l, h)$ plane for selected design parameters

Fig. 4 shows the path of the water jet flowing from the nozzle at an angle of $\alpha$ determined on the basis of selected design parameters of the fountain: $D_{\text {na }}=0.29 \mathrm{~m}, \quad \chi=0.5 \quad\left(D_{\mathrm{c}}=0.145 \mathrm{~m}\right), \quad H=h_{\text {max }}=$ $4 \mathrm{~m}$, and $\sigma=0.5\left(H_{\mathrm{c}}=2 \mathrm{~m}\right)$. For this case, equation (16) becomes:

$$
0.0158 \tan ^{2} \alpha-2.0092 \tan \alpha+32=0
$$

and the angle $\alpha$ determined from equation (19) or (20) have values:

$$
\begin{array}{ll}
\alpha_{1}=1.517256 \mathrm{rad} & \alpha_{1}=86.93234^{\circ} \\
\alpha_{2}=1.561602 \mathrm{rad} & \alpha_{2}=89.47318^{\circ}
\end{array}
$$




\subsection{The diameters of the fountain image of hourglass type.}

The basic parameters of the fountain include: its radius $R_{\mathrm{f}}$ which is measured at ground level and radius $R_{\max }$ which specifies the distances of the $n$ jets tops the axis passing through the point $\mathrm{C}$ (the centre of the fountain). Both of these parameters are dependent on the basic construction parameters, i.e.. $R_{\mathrm{f}}=f\left(D_{\text {na }}, \chi, H, \sigma\right)$ and $R_{\text {max }}=f\left(D_{\text {na }}, \chi, H, \sigma\right)$.

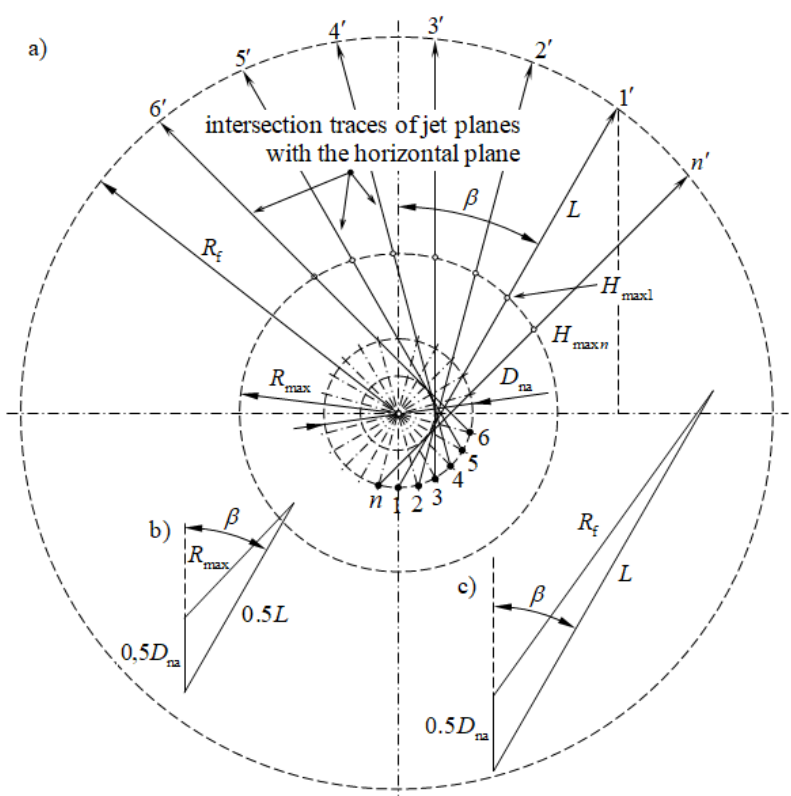

Fig. 5. Top view of fountain - basic dimensions

Fig. 5 presents a fountain diagram in a horizontal view. In this drawing both parameters discussed above are visible. To determine the $R_{\max }$ and $R_{\mathrm{f}}$ ancillary diagrams were prepared, respectively b) and c). All $n$ traces have a length $L$ defined by formula (7). To this formula was substituted square of initial velocity $v_{0}^{2}=2 g h_{\max } / \sin ^{2} \alpha$ designated by the formula (15). Formula defining the range of the stream, therefore takes the form

$$
L=\frac{4 h_{\max } \sin \alpha \cos \alpha}{\sin ^{2} \alpha}=4 h_{\text {max }} \tan ^{-1} \alpha
$$

After substituting for the equation (21) value of previously determined angle $\alpha$, (the range of each stream, which is the part of the fountain), is specified by a formula

$$
\begin{aligned}
L & =4 h_{\max } \tan ^{-1}\left(\arctan \frac{4(1 \pm \sqrt{1-\sigma}) h_{\max }}{D_{n} \sqrt{1-\chi^{2}}}\right) \\
& =\frac{D_{n} \sqrt{1-\chi^{2}}}{1 \pm \sqrt{1-\sigma}}
\end{aligned}
$$

Based on Fig. 5b), after using the cosine theorem can be written

$$
R_{\max }=\sqrt{\left(0.5 D_{\mathrm{n}}\right)^{2}+(0.5 L)^{2}-0.5 D_{\mathrm{n}} L \cos \beta}
$$

After substituting $\cos \beta=\sqrt{1-\chi^{2}}$ and the right side of formula (22) for $L$, after suitable transformations we obtain

$$
R_{\max }=0.5 D_{\mathrm{n}} \sqrt{1+\frac{1-\chi^{2}}{(1 \pm \sqrt{1-\sigma})^{2}}-\frac{2\left(1-\chi^{2}\right)}{1 \pm \sqrt{1-\sigma}}}
$$

Similarly, on the basis of Fig. 5c) using the cosine theorem can write

$$
R_{\mathrm{f}}=\sqrt{\left(0.5 D_{\mathrm{n}}\right)^{2}+L^{2}-D_{\mathrm{n}} L \cos \beta}
$$

Again after substituting for $\cos \beta$ and $L$ we obtain

$$
R_{\mathrm{f}}=0.5 D_{\mathrm{n}} \sqrt{1+\frac{4\left(1-\chi^{2}\right)}{(1 \pm \sqrt{1-\sigma})^{2}}-\frac{4\left(1-\chi^{2}\right)}{1 \pm \sqrt{1-\sigma}}}
$$

For the numerical data of the design parameters used for drawing Fig. $4 \quad(\chi=0.5, \sigma=0.5$ value of $R_{\mathrm{f}}=0.735 \mathrm{~m}$ was obtained).

\section{Simulation of a fountain picture}

Fig. 6 shows a fountain diagram (horizontal projection) illustrating geometrical relationships allowing the simulation of a fountain image.

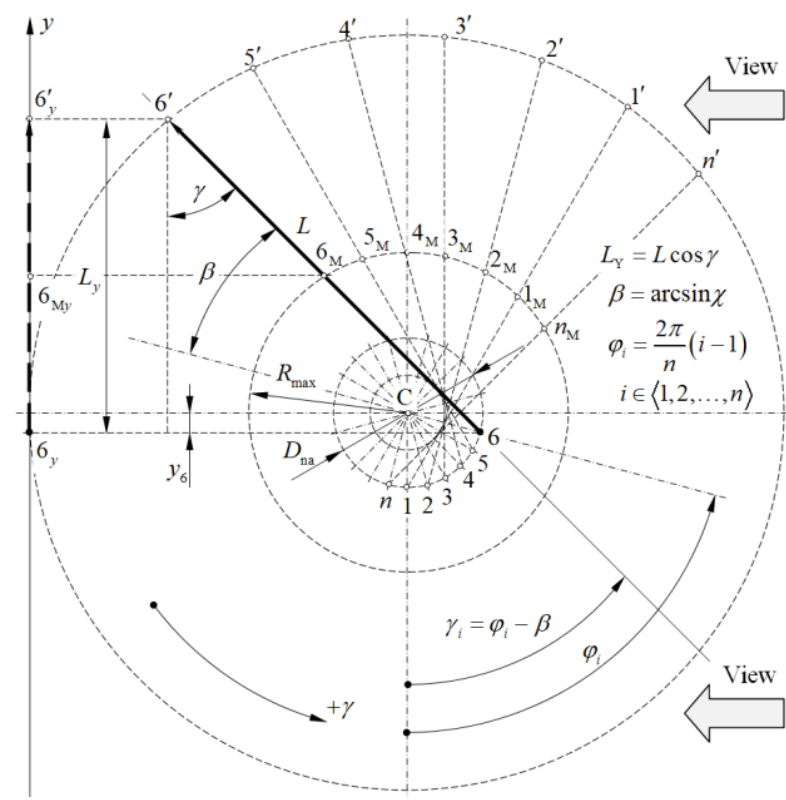

Fig. 6. Diagram used to determine the basic parameters of the simulation

The simulation was carried out in a few steps

- determine the angle $\varphi_{\mathrm{i}}$

Angle $\varphi_{\mathrm{i}}$ is measured from the straight line connecting the centre of the fountain $\mathrm{C}$ with the axis of the first nozzle $n=1$ in counter clockwise direction, it is determined by the relation:

$$
\varphi_{i}=\frac{2 \pi}{n}(i-1) \quad \mathrm{rad}
$$


- determination of the $y_{i}$ coordinate of the position of the vector origin $L_{y}$ in the axis $\mathrm{y}-$ e.g. point $6_{y}$ coordinate in Fig. 6

$$
y_{i}=-0.5 D_{\mathrm{na}} \cos \varphi_{i} \quad \mathrm{~m}
$$

Minus in formula (27) follows from the fact that the nozzle 1 is located in the negative portion of the axis $y$.

- determination of the angle $\gamma_{i}$, which determines the deviation of the trace of the stream flow surface from the straight line $\overline{\mathrm{C}-1}$.

$$
\gamma_{i}=\varphi_{i}-\beta \text { where } \beta=\arcsin \chi
$$

- determining of jest horizontal projection length $L$ and its division into $j$ calculation points. The simulation presented in the paper was based on $m=41$ calculation points. Therefore, the coordinate of the calculation points is

$$
l_{j}=\frac{L}{m} j
$$

- determination of calculation points coordinates on the axis $y$.

$$
l_{j y}=y_{i}+l_{j} \cos \gamma_{i}
$$

where coordinate $y_{i}$ is constant for a given jet (27), e.g. for $i=6, y_{6}=-0.0375 \mathrm{~m}$.

- creation of a matrix consisting of $m$ rows (the number of calculation points for each stream), and $n$ columns (number of nozzles) on the basis of formula (31).

- to the each column should be assigned values $h_{i}=f\left(l_{i y}\right)$, which will be visible on the chart. It was the most time-consuming step of simulation preparing. After the introduction of the first pair of series of data, there was created a graph illustrating the course of the value projection e.g. stream from the nozzle 1 on the plane of the desired view. Then you had to choose a chart, go to the Chart Tools on the command bar to the Design tab and choose the Select Date (Fig. 7).

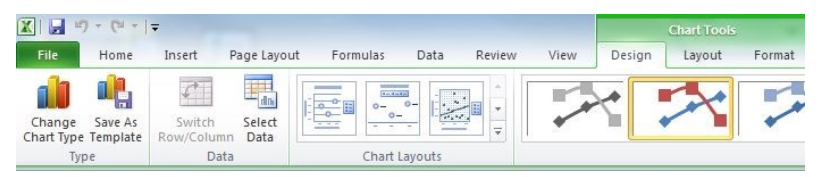

Fig. 7. Chart Tools bar with a button Select Data

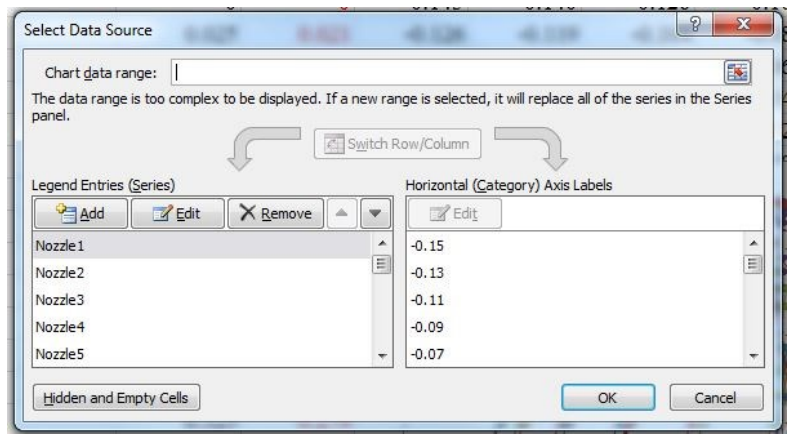

Fig. 8. Dialogue window Select Data Source
This causes calling the Select Data Source dialog. If you want to add another pair of data series should in the window Legend Entries (Series), click the Add button.

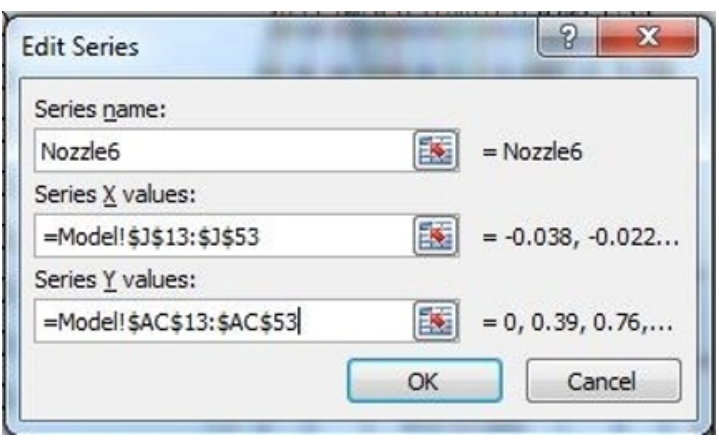

Fig. 9. Dialogue window Edit Series

This action causes the Edit Series dialog box. It differs from the window shown in Fig. 9 that all the fields are empty. The window that appears when you click the Add Series, is the same as when you click Edit Series.

For all nozzles, field Series Y values was filled with the same data including horizontal input data ( $y$ axis in Fig. 10).

\section{Conclusions}

- From the practical point of view, the most significant is the formula (19) allowing to determine the angle of inclination of the nozzle to the ground horizontal level $\alpha$, i.e. the basic parameter which defines the angle of outflow of the nozzle, ensuring achievement of the assumed construction parameters of the fountain.

- The formulas (24) and (26) allow calculation the diameter of the fountain $D_{\mathrm{f}}$ and diameter of tops jets distribution, in Fig. 4 named as $\mathbf{M}_{i}$. It may seem surprising that the design parameter $h_{\max }$ does not exist in these formulas. However, it is hidden in the factor $\sigma$.

- Described simulation enables rapid image analysis of water fountains, which is important from the point of view of the manufacturer fountains negotiations with the client. Fountains sample image shown in Fig. 10, Fig. 11, Fig. 12, and Fig 13.

- Fig. 10 shows the fountain picture in a scale of length design parameters sizes similar to the actual dimensional ratio. $h / l \approx 1$. Time to the new image fountains, including to enter of new parameters is measured in seconds.

- Figs 11, 12, 13 are presented in a reduced height scale $h / l \square \quad$.

- Shortly before this work was written, preliminary tests were carried out. The result seems to confirm the theory of the model, see Fig. 14. However, with the final conclusion we should wait for the device to be made, which allows the exact $\alpha$ angle setting.

Acknowledgement: The authors would like to kindly thank the company KORRO PLUS, Bydgoszcz, Poland for cooperation in the implementation of the project. Authors also received 
institutional support BS 28/2018 granted by Faculty of Mechanical Engineering of UTP University.

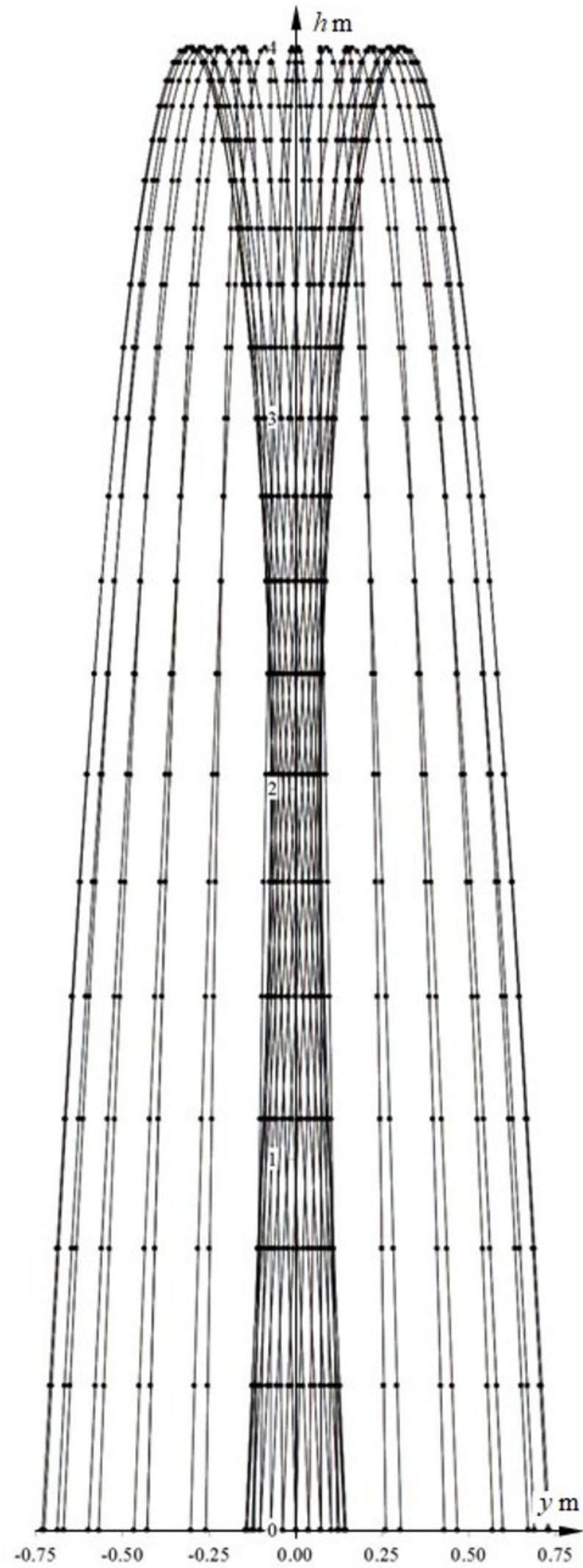

Fig. 10. Simulation effect for $H=4 \mathrm{~m}, D_{\mathrm{na}}=0.29 \mathrm{~m}$, $\chi=0.5$, and $\sigma=0.5$

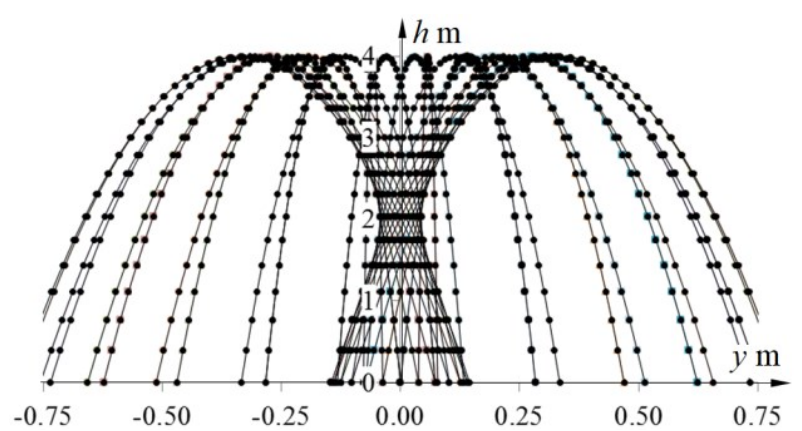

Fig. 11. Simulation effect for $H=4 \mathrm{~m}, D_{\mathrm{na}}=0.29 \mathrm{~m}$, $\chi=0.3$, and $\sigma=0.5$

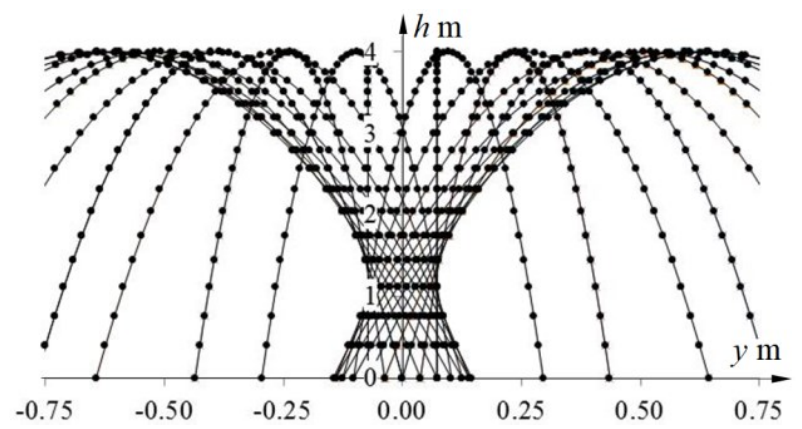

Fig. 12. Simulation effect for $H=4 \mathrm{~m}, D_{\text {na }}=0.29 \mathrm{~m}$, $\chi=0.5$, and $\sigma=0.3$

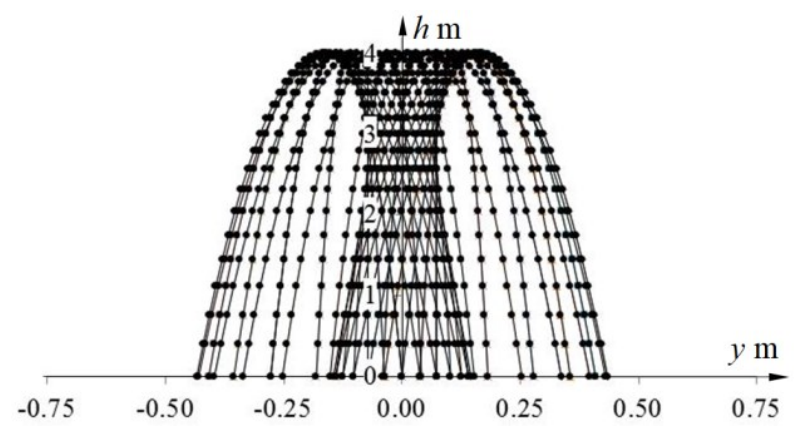

Fig. 13. Simulation effect for $H=4 \mathrm{~m}, D_{\mathrm{na}}=0.29 \mathrm{~m}$, $\chi=0.5$, and $\sigma=0.7$

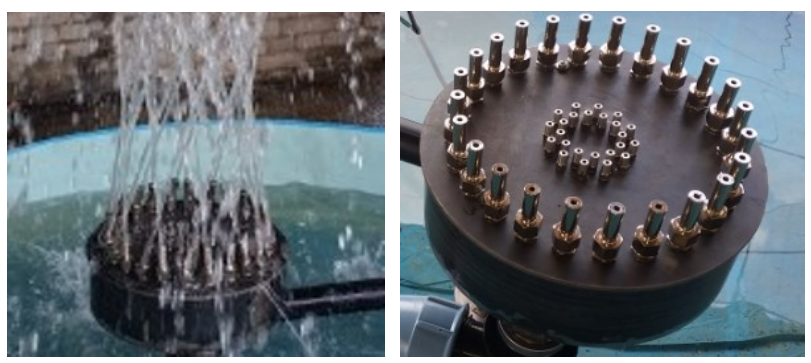

Fig. 14. (Left) Very initial test results. (Right) Assemble of nozzles - the paper treats the outer set.

\section{References}

1. Y.A Cengel, J.M Cimbala: Fluid Mechanics. Fundamentals and Applications, McGraw-Hill, (2006) 